Osorio R, Sauro S, Watson TF, Toledano M. Polyaspartic acid enhances dentine remineralization bonded with a zinc-doped Portland-based resin cement. Int Endod J. 2015 Aug 13. doi: 10.1111/iej.12518.

Polyaspartic acid enhances dentine remineralization bonded with a zinc-doped Portland-based resin cement.

Osorio $\mathrm{R}^{\mathrm{a}^{*}}$, Sauro $\mathrm{S}^{\mathrm{b}}$, Watson $\mathrm{TF}^{\mathrm{c}}$, Toledano $\mathrm{M}^{\mathrm{a}}$

a - Dental Materials, School of Dentistry, University of Granada, Colegio Máximo, Campus de Cartuja, Granada, Spain.

b - Dental Biomaterials and Minimally Invasive Dentistry, Departamento de Odontología, Facultad de Ciencias de la Salud, CEU-Cardenal Herrera University, Valencia, Spain.

c - Biomaterials, Biomimetics \& Biophotonics, King's College London Dental Institute at Guy's Hospital, London, UK.

Running title: Polyaspartic acid enhances dentine mineralization

Key words: Bonding, Dentine, Remineralization, Resin.

ACKNOWLEDGMENTS: This work was supported by grant MINECO/FEDER MAT2014-52036-P.

\title{
*CORRESPONDING AUTHOR
}

Prof. Raquel Osorio.

School of Dentistry, Colegio Máximo.

Campus de Cartuja s/n.

University of Granada

E-18071 Granada, Spain.

E-mail: rosorio@ugr.es 


\section{ABSTRACT}

Aim. To ascertain if biomimetic phosphoproteins analogues (polyaspartic acid -PAS- and sodium trimetaphosphate -TMP-) improve bonding efficacy and dentine remineralization ability of a novel zinc-doped Portland-based resinous sealing cement.

Methodology. Bonding procedures were performed on phosphoric acid etched dentine and several groups were established regarding biomimetic analogue application: 1) No application, 2) PAStreated dentine and 3) dentine treated with a mixture of PAS and TMP. Raman spectroscopy and microtensile bond strength (MTBS) with fracture analysis by scanning electron microscopy were carried out. MTBS values were compared by ANOVA, Student-Newman-Keuls and Student $t$ tests $(\mathrm{P}<0.05$ and $\mathrm{P}<0.01$ respectively).

Results. MTBS values were not affected by the different bonding procedures, at $24 \mathrm{~h}$ testing. After 6 months, MTBS decreased in those groups in which analogues phosphoproteins' were not applied $(P<0.05)$. When Pas was applied, MTBS was maintained after 6 months $(P>0.05)$. The novel material bonded without primers application induced bioactive crystal (calcium carbonate and Ettringite) precipitation onto the etched dentine and augmented the degree of crystallinity at the hybrid layer. Mineral to matrix ratio was increased at the hybrid layer of the PAS-treated specimens; this primer was also able to catalyze dentine remineralization, without an increase in crystallinity.

Conclusions: PAS application onto demineralized dentine produced an inhibition or delay of the mineral phase crystallization, enhancing the remineralization potential of the Portland microfillers at the resin-dentine bonded interface. 


\section{INTRODUCTION}

New materials for use in endodontics, including root-end filling, root-perforation repair or regeneration should facilitate dentine remineralization and inhibit collagen degradation inhibition at the dentine interface (Gandolfi et al., 2010a, Gandolfi et al./ 2010b, Osorio et al. 2014). Components such as calcium/sodium phosphosilicate, tricalcium silicates, tricalcium phosphate and zinc oxide have been included within the composition of resin-based canal sealing materials to create bioactive cements. These materials can facilitate sealing, metalloproteinases inhibition and tissue remineralization (Gandolfi et al./ 2010a, Dorozhkin 2010, Osorio et al. 2014). Their application in root canal treatments is recently being investigated and encouraged (Gandolfi et al./ 2010b, Osorio et al. 2012, Osorio et al./ 2014).

Non-collagenous proteins play a critical role to orchestrate dentine mineralization. They possess carboxylic and phosphate functional groups that act as sites for calcium and phosphate nucleation (Liu et al. 2011). Portland-based cements are likely to be able to remineralize partially demineralized dentine by epitaxial deposition of calcium and phosphate phases over remnant apatite seed crystallites (Watson et al. 2014). Although these materials release silicon, calcium and phosphate, they lack biomimetic analogs to sequester those ions into amorphous calcium phosphate prenucleation clusters. As crystalline calcium phosphates and silicates have long degradation times, in the order of months or even years (Rezwan et al. 2006), it is difficult to envisage how the use of these materials can remineralize collagen fibrils within a faulty hybrid layer.

Application of synthetic substitutes for dentine matrix proteins has been proposed to facilitate remineralization processes (Sauro et al. 2015). Two different dentine analogous phosphoproteins' may be needed: i) a sequestering agent that controls amorphous calcium phosphate aggregation and ii) a phosphoproteins substitute that will facilitate positioning of hydroxyapatite at specific sites on collagen, templating the nucleation of precursors at the special sites of the collagen substrate (Gu et al. 2011, Watson et al. 2014). Polyaspartic acid and sodium trimetaphosphate possess functional groups (i.e. carboxylic and phosphate), and may have the ability to sequester and to release calcium 
and phosphate, in order to adapt to the demands of the developing mineral, probably acting as phosphoproteins biomimetic analogs (Gu et al. 2011, Liu et al. 2011, Sauro et al. 2015). These amorphous compounds may provide a local ion-rich environment that will be considered favorable for dentine remineralization (Gu et al. 2011, Liu et al. 2011).

The aim of this study was ton test the bonding efficacy and remineralization ability of a novel ionreleasing resin material bonded with or without biomimetic analogues phosphoproteins' (polyaspartic acid -PAS- or a combination of PAS with sodium trimetaphosphate) at the dentine interface. The null hypothesis is that application of two biomimetic analogues phosphoproteins' on etched dentine does not influence bond strength and dentine remineralization ability, when bonding with a resin-based experimental endodontic cement, containing bioactive particles.

\section{MATERIALS AND METHODS}

Material formulation and bonding procedures: A zinc-doped Portland-based resin cement was formulated using urethane dimethacrylate (30 wt\%), ethoxylatedbisphenol-A dimethacrylate (5 wt\%), triethyleneglycoldimethacrylate (25 wt\%) (Esstech, Essington, PA, USA), hydroxyethyl methacrylate (18wt\%), absolute ethanol (15 wt\%), camphorquinone (1.0 wt\%) and ethyl-4dimethylaminobenzoate (1.0 wt\%) (Sigma-Aldrich, St. Louis, MO, USA). For filler production, a type I Portland cement (Italcementi Group, Bergamo, Italy) (70 wt\%) was mixed with 10 wt\% $\beta$-TCP (SigmaAldrich), 20 wt\% zinc oxide particles (Sigma-Aldrich) and deionized water (ratio 2:1). After setting $(24 \mathrm{~h})$, the cement was incubated at $40^{\circ} \mathrm{C}$ for $12 \mathrm{~h}$, milled, sieved to $<30 \mu \mathrm{m}$ and incorporated as a filler into the unfilled resin control (filler/resin ratio: $40 / 60 \mathrm{wt} \%$ ) to create the experimental ionreleasing resin (BTCS-Zn) (Sauro et al. 2013, Osorio et al. 2014).

Two biomimetic solutions were tested: 1) $150 \mu \mathrm{g} / \mathrm{mL}$ of poly-L-aspartic acid (PAS) with a molecular weight of $27 \mathrm{kDa}$ (Sigma-Aldrich) solvated in deionized water, and 2) $150 \mu \mathrm{g} / \mathrm{mL}$ of PAS in deionized water mixed with 10 wt\% sodium trimetaphosphate (molecular weight of $367.8 \mathrm{kDa}$ ) (TMP; SigmaAldrich). The pH of both solutions was adjusted to 7.1 (Sauro et al. 2015). 
Human third molars were obtained with informed consent from donors and used in accordance to the Research Ethics Committee. Dentine was polished (320-grit) and after acid-etching $\left(37 \% \mathrm{H}_{3} \mathrm{PO}_{4}\right.$; 15 s) the specimens were pre-treated by immersion in deionized water (DW) or using each biomimetic solution PAS or PAS-TMP, for $1 \mathrm{~min}$. These specimens were bonded with two consecutive layers of the ion-releasing resin cement. Polymerization was performed and the light was tested for light output $\left(600 \mathrm{~mW} / \mathrm{cm}^{2}\right)$ by means of a Demetron radiometer (Model 100, Demetron Research Corp., Danbury, CT, USA) (Sauro et al. 2013).

Raman spectrometer analysis: Three bonded interfaces per group were created and submitted to a dispersive Raman spectrometer analysis (Horiba Scientific Xplora, Villeneuve d'Ascq, France), after $24 \mathrm{~h}$ and 1 month of artificial saliva (AS) storage. The composition of artificial saliva was $0.7 \mathrm{mM} / \mathrm{L}$ $\mathrm{CaCl}_{2}, 0.2 \mathrm{mM} / \mathrm{L} \mathrm{MgCl}_{2}, 4.0 \mathrm{mM} / \mathrm{L} \mathrm{KH}_{2} \mathrm{PO}_{4}, 30.0 \mathrm{mM} / \mathrm{L} \mathrm{KCl}, 20.0 \mathrm{mM} / \mathrm{L}$ HEPES (Sauro et al. 2015). For Raman analysis, a 785-nm diode laser (100 mW sample power) equipped with a $\times 100 / 0.90 \mathrm{NA}$ water-immersion objective was employed. Raman signals were acquired using a 600 -lines $/ \mathrm{mm}$ grating centered between 900 and $1700 \mathrm{~cm}^{-1}$. Three chemical mappings were captured along the interfaces and submitted to K-means cluster analysis as described by Toledano et al. (2014a, 2014b), using multivariate analysis (ISyss Horiba, Horiba Scientific Xplora, Villeneuve d'Ascq, France), which includes statistical patterning to derive independent clusters; the biochemical content of each cluster was analyzed using the average cluster spectra. Principal component analysis decomposed data were set into a bilinear model of linear independent variables, the so-called principal components. Four clusters were identified (adhesive, hybrid layer, dentine 1 and dentine 2) [dentine 1 and 2 are related to dentin with different degrees of mineralization]. At this point, in order to quantitatively calculate the extent of mineralization along the interface, the following parameters were measured for the same clusters in each group: 1) the mineral matrix ratio (MMR: the relative mineral content, or the degree of demineralization as a function of spatial position, which was determined from the ratios of the relative integrated intensities of spectral features associated with phosphate ( $\mathrm{P}-\mathrm{O}$ symmetric stretch: $\left.v_{1}-961 \mathrm{~cm}^{-1}\right)$ and collagen $\left(\mathrm{CH}_{2}\right.$ deformation: $\left.\left.1454 \mathrm{~cm}^{-1}\right) ; 2\right)$ the relative presence of mineral (RPM: the phosphate peak height $\left(v_{1}-961 \mathrm{~cm}^{-1}\right)$ (Wang et al. 2009); and 
3) the degree of crystallinity (FWMH: the full width at half maximum of the phosphate band $\left(v_{1}{ }^{-}\right.$ $961 \mathrm{~cm}^{-1}$ ), as it expresses the crystallographic or relative atomic order; the narrower the spectral peak width, the higher the degree of mineral crystallinity (Karan et al. 2009, Toledano et al. 2014a). Microtensile Bond strength testing (MTBS): A further six resin-bonded dentine specimens were prepared for each group and sectioned to obtain multiple bonded-sticks of $0.9 \mathrm{~mm}^{2}$. Their microtensile bond strengths were tested after $24 \mathrm{~h}$ or $6 \mathrm{~m}$ of AS storage using a customized microtensile jig on a linear actuator (SMAC Europe Ltd., Horsham, UK). Mean bond strength values were analyzed by ANOVA, which was performed including the bond strength (MPa) as the dependent variable. Bonding groups (DW, PAS or PAS-TMP) and period of AS storage ( $24 \mathrm{~h}$ or 6 months) were considered as independent variables. Analysis of interactions was also conducted. Student-Newman-Keuls and Student $t$ tests were used for comparisons. Statistical analyses were set at a significance level of $\alpha=0.05$ and $\alpha=0.01$ respectively. Modes of failure were classified as percentage of adhesive (A) or mixed (M) or cohesive (C) failures using a stereo microscope (magnification $\times 60$ ). Three representative fractured specimens for each group were mounted on aluminium stubs using carbon tape, carbon-sputter-coated and finally imaged using a field emission scanning electron microscopy SEM (Gemini, Carl Zeiss, Oberkochen, Germany) at 3 kV and a working distance of 6 to $7 \mathrm{~mm}$. The microscope was attached to an energy dispersive analysis system (EDX) (Inca 300 and 350, Oxford Instruments, Oxford, UK).

\section{RESULTS}

Results from Raman analysis are presented in Table 1 and Figures 1 and 2. Mineral to matrix ratios at the hybrid layer, were different regarding the distinct primers application. When no biomimetic solution was used before bonding procedures, the MMR did not change, being 1.68 and 1.56 at base line and after 1 month, respectively. When PAS was applied on etched dentine, the MMR at the hybrid layer presented a 7.5-fold augmentation (from 0.72 to 5.40 ) and when PAS-TMP was applied, it presented a 2 -fold increase (from 1.24 to 2.55). These changes may also be appreciated on the phosphate peak intensity graphs, where a patent augmentation of phosphate peak intensity (green 
changes into red) is observed at the underlying dentine; this occurred mainly inside the dentinal tubules (Figures 1a, 1d, 1g, 2a, 2d and 2g). The absence of the dentinal tubular appearance was also detected after 1 month of storage, in PAS and PAS-TMP groups (Figures $2 \mathrm{~d}$ and $2 \mathrm{~g}$ ). On the cluster analysis image, the hybrid layer (blue) was clearly diminished in thickness at PAS/BTCS-Zn specimens after 1 month of storage (Figure 2e).

After 1 month, the degree of crystallinity in the existing mineral at the interface was higher (2-fold increase) when the ion-releasing adhesive was employed without PAS application. The width of the phosphate peak at the hybrid layer was about 38.74 after $24 \mathrm{~h}$ and diminished to 20.52 (increasing in sharpness) after 1 month. The same trend was observed in the underlying dentine. However, when PAS was applied, the degree of crystallinity in the new mineral at the interface is reduced. At the hybrid layer, peak changes in width were from 35.33 to 45.72 and in the underlying dentine from 28.93 to 38.52 (Table 1 ).

On DW/BTCS-Zn specimen spectra, bands indicating relative presence of organic components of type I collagen $\left(1,245 ; 1,465 ;\right.$ and 1,667 $\left.\mathrm{cm}^{-1}\right)$ and hydroxyproline $\left(921 \mathrm{~cm}^{-1}\right)$ were decreased after 1 month storage. After 1 month storage, a band $\left(1,000 \mathrm{~cm}^{-1}\right)$ corresponding to the internal $\mathrm{PO}_{4}{ }^{3-} v_{1}$ vibration mode in zinc phosphate compounds, was encountered (Figures 1c and 2c).

On PAS-TMP/BTCS-Zn spectra after $24 \mathrm{~h}$, the highest intensity of the band at $1,465 \mathrm{~cm}^{-1}$ was observed in the adhesive layer. A distinct peak around 1,100 and $1,150 \mathrm{~cm}^{-1}$ appeared, after 1 month, this band was associated to the presence of polyphosphates compounds (Figures 1i,2i).

Hybrid layer spectra contained features of both polymers and dentine spectra, except for the hybrid layer spectrum from PAS/BTCS-Zn specimens, after 1 month of storage which resembles almost completely a characteristic dentine spectrum, but with less mineral content (Figure 2f).

Bond strength was affected by bonding procedure $(P<0.05)$, by storage time $(P<0.01)$ and the interactions were also significant $(P<0.01)$. The power of the ANOVA was 0.70 . Mean bond strengths are depicted in Table 2. At $24 \mathrm{~h}$, bond strength values attained by the experimental adhesive were similar for all groups, regardless of the different biomimetic analogue applications. After 6 months, a 
significant decrease in the bond strength values was observed, except for the group in which dentine was pre-treated only with PAS (34.0 MPa); this value was significantly higher than those obtained when no biomimetic analogues were used (24.1 MPa) or after PAS-TMP application (24.4 MPa) $(\mathrm{P}<0.05)$. Debonding was most prevalent in mixed mode (95 to 90\%) (Table 2).

After 6 months, zones where resin was covering collagen and mineralized resin tags were observed on the SEM images performed on those specimens bonded without PAS (Figure 3a). Other zones exhibit demineralized and not resin infiltrated collagen with some resin remnants located mainly inside the dentinal tubules (Figure $3 b$ ). On these specimens, mineral precipitation was observed. Spherical mineral formations containing calcium, silicon and aluminum (Figure 3c) and needle shape crystals (Figure 3d) composed of zinc, calcium and silicon, were seen. On those specimens bonded after PAS application, no demineralized collagen was observed. Intertubular dentine was hardly distinguished. Fractured resin tags were present inside the dentine tubules and mineral precipitates were present (Figures $3 e$ and $3 f$ ). Those specimens treated with PAS-TMP had both highly mineralized broken resin tags with remineralized peritubular dentine and areas in which demineralized collagen remained unprotected (Figures 3g, 3h and 3i).

\section{DISCUSSION}

Pre-treatment of acid-etched dentine using PAS and PAS-TMP improved the remineralization ability of an experimental light-curable resin-based system containing zinc-doped Portland micro-fillers.

In the present study, resin-based materials, including reactive calcium-phospho-silicate powders as fillers, would be able to release mineral ions and produce bioactive crystals formation, at the bonded dentine interface. When no primer was applied before bonding, hexacalcium aluminate trisulfate (Ettringite) was found at the dentine surface, after resin debonding (Figure 3d-Ep2-). Ettringite in Portland or Mineral Trioxide Aggregate cements is formed when calcium alumina silicate crystals are in contact with water and undergo a hydration process (Camilleri 2008). On the present EDX spectrum Zn was also present, and the Ettringite crystal systems were elongated in a needle like 
shape and the crystal morphology could incorporate a variety of divalent ions (i.e. $\mathrm{Zn}^{2+}$ ), which can substitute for $\mathrm{Ca}^{2+}$ by incorporating these ions into the lattice (Moon et al. 2013). When $\mathrm{Ca}^{2+}$ and $\mathrm{OH}^{-}$ ions are released from tricalcium silicate into the surrounding environment at supersaturation levels, a calcium hydroxide (portlandite) precipitate, and in the presence of carbon dioxide this may also precipitate as calcium carbonate (Camilleri 2008, Parirokh\&Torabinejad 2010). Rounded calcium carbonate crystals were also detected at the debonded resin-dentine interface (Figure 4c-Ep1-); calcium carbonate and calcium hydroxide have been previously identified as hydroxyapatite precursors (Watson et al. 2014). Calcium carbonate $\left(1,071 \mathrm{~cm}^{-1}\right)$ was also identified, after 1 month, in the Raman analysis of the bonded interface at DW/BTCS-Zn specimens (Figure 2c).

These crystal formations were not encountered in any of the other experimental groups, in which PAS was applied. PAS is a biodegradable, water-soluble polyaminoacid with potential to inhibit deposition of calcium carbonate and calcium phosphate salts (Hassonet al. 2011). PAS molecules become adsorbed on the collagen fibres, and retarded the mineralization process. The mineralization of collagen is highly dependent upon the interaction of collagen with such acidic molecules. PAS application will guarantee that crystal precipitation will not occur, and calcium and phosphate may remain available to diffuse into the collagen of dentine. Ion stabilization is considered as a key factor, to allow remineralization of demineralized dentine (Liuet al. 2011, Li et al. 2013). These results are in accordance with the Raman analysis. On Raman spectra, after 1 month of storage, the degree of crystallization was increased (2-fold) when the ion releasing adhesive was employed without PAS application (Figure $2 \mathrm{c}$ and Table 1). However, when PAS was applied onto etched dentine, degree of crystallinity was reduced (Figure $2 f$ and Table 1 ). This is an important finding, as one of the main problems when applying inorganic remineralizing materials, is the poor solubility of the calcium phosphate phases at the normal pH range of biological oral fluids (Cochrane et al. 2010). Even, if unstabilised amorphous calcium phosphates and silicates are applied they will tend to be transformed into a stable crystalline phase (Cochrane et al. 2010). Therefore, If PAS application onto dentine is able to delay or inhibit phase crystallization; it will directly improve the remineralizing ability of calcium silicate cements. 
Mineral to matrix ratio in the underlying dentine, increased equally after 1 month storage in all groups (Table 1). This confirms the remineralizing effect of these modified-Portland fillers (Parirokh \& Torabinejad 2010, Watson et al. 2014). In the phosphate peak intensity graphs (Figure 1 and 2), a clear augmentation of phosphate peak intensity (green changes into red) is observed in the underlying dentine. This occurred mainly inside the dentinal tubules (appreciated by the graphical representation of the clusters distribution), the absence of the dentinal tubular appearance after 1 month of storage is observed in the PAS and PAS/TMP groups (Figures $2 \mathrm{e}$ and $2 \mathrm{~h}$ ). These findings were also observed on the SEM images, as intratubular and peritubular remineralization was present in all groups (Figure $3 \mathrm{a}, 3 \mathrm{f}$ and $3 \mathrm{~h}$ ). After dentine bonding, the major fluid content existed inside dentinal tubules, which explains the presence of abundant mineral precipitation when using these cements (Watson et al. 2014). However, this remineralization does not guarantee collagen protection in the hybrid layer. When analyzing the Raman spectra on the hybrid layer the mineral to matrix ratio changed as a function of the distinct primer application. When PAS was applied on etched dentine, the MMR in the hybrid layer increased 7.5-fold and when PAS-TMP were applied it increased 2 -fold and remained at similar values when no primers were applied (Table 1 ). This confirms the relevance of PAS application on etched dentine. The presence of amorphous calcium and phosphate compounds will lead to remineralization of the hybrid layer. After 1 month, only the Raman spectra on the hybrid layer in those specimens treated with PAS were similar to that of dentine (even when it revealed lower mineral content)(Figure 2f). This suggests that PAS plays a critical role in the hybrid layer recovering the properties of dentine.

Acid-etched dentine surfaces pretreated with PAS and bonded with the experimental adhesive maintained MTBS values after 6 months. Higher bond strength values were attained when PAS was applied without TMP. These findings may be explained by: i) TMP may produce alterations within the resin polymer on the Raman spectra the band at $1,465 \mathrm{~cm}^{-1}\left(\mathrm{CH}_{2}\right.$ asymmetric bending from methacrylate) was higher when compared to the other groups (Figure 1i). This may be related with its retarded polymerization (Zhang \&Wang 2013). ii) Zinc in the filler may form polyphosphate 
complexes with TMP, and may interfere with the desired effect of this compound (Rashchi \& Finch 2000, Cini \& Ball 2014). A distinct peak between 1,100 and 1,150 $\mathrm{cm}^{-1}$ appeared, after 1 month, and this corresponds to the polyphosphates salts formed (Figure 2i) (Omelonet al. 2014). In those dentine specimens bonded without biomimetic primers or with PAS-TMP, areas with demineralized and non-resin infiltrated collagen fibrils were observed (Figures $3 \mathrm{~b}$ and $3 \mathrm{i}$ ), even when tubules were mostly occluded by mineral and/or crystals (Figures 3c, 3d and 3h).

TMP was shown to have the potential for phosphorylating type I native collagen, but when TMP has been previously used in dentine, the degree of remineralization was not as expected and collagen degradation occurred after 4 months (Liu et al. 2011). TMP is a polyphosphate that is slightly soluble in water $(22 \mathrm{~g} / 100 \mathrm{~mL})$, and protein phosphorylation with TMP usually requires a $\mathrm{pH}>11$ (Shen 1966). Moreover, polyphosphates have been shown to inhibit mineralization as they competitively saturate alkaline phosphatase, which is present in dentine (but not in native collagen), thus, potentially interfering with alkaline phosphatase ability to hydrolyze mineralization-inhibiting pyrophosphate (PPi)(Omelon et al. 2014, Hoac et al. 2013). So, other different phosphate compounds, such as dipotassium phosphate, that is soluble in water $(149.25 \mathrm{~g} / 100 \mathrm{~mL})$ and is not a polyphosphate, are recommended for dentine remineralization (Burwell et al. 2012).

The presence of other ions may also be important to explain the results. Silicon is known to facilitate calcium phosphate deposition (Leonor et al. 2009), and plays a critical role on the binding of calcium phosphate complexes to the collagen network (Besinis et al. 2014). Silicon has been shown to mediate the formation of calcium phosphate precursors, necessary for the subsequent steps of mineralization, acting also as a nucleating mineral (Watson et al. 2014). It may be that the same mechanism of cooperative nucleation occurs on dentine collagen with PAS and silicon ions, not requiring TMP application.

Pure Portland and MTA cements containing tri-calcium and di-calcium silicate (which on hydration produce calcium silicate hydrate gel, calcium hydroxide, and calcium carbonate) are able to exert remineralizing effects (Parirokh \& Torabinejad 2010, Watson et al. 2014). If PAS should also increase this potential when in contact with dentine, deserves further research. 


\section{CONCLUSIONS}

i) Portland microfillers incorporated into resin-based cements are able to set, forming bioactive crystals (calcium carbonate and calcium aluminate sulfate), preferentially located, inside dentinal tubules;

ii) PAS application onto demineralized dentine inhibited or delayed crystallization. The presence of amorphous compounds increased the remineralization potential of the Portland microfillers at the resin-dentine bonded interface. 


\section{REFERENCES}

Besinis A, van Noort R, Martin N (2014) Remineralization potential of fully demineralized dentin infiltrated with silica and hydroxyapatite nanoparticles.Dental Materials 30, 249-62.

Burwell AK, Thula-Mata T, Gower LB et al. (2012) Functional remineralization of dentin lesions using polymer-induced liquid-precursor process. PLoS One 7, e38852.

Camilleri J (2008) Characterization of hydration products of mineral trioxide aggregate. International Endodontics Journal 1, 408-17.

Cini N, Ball V (2014) Polyphosphates as inorganic polyelectrolytes interacting with oppositely charged ions, polymers and deposited on surfaces: fundamentals and applications. Advances in Colloids and Interface Science 209, 84-97.

Cochrane NJ, Cai F, Huq NL, Burrow MF, Reynolds EC (2010) New approaches to enhanced remineralization of tooth enamel. Journal of Dental Research 89, 1187-97.

Dorozhkin SV (2010) Bioceramics of calcium orthophosphates. Biomaterials 31, 1465-85.

Gandolfi MG, Ciapetti G, Taddei P, Perut F, Tinti A, Cardoso MV, Van Meerbeek B, Prati C (2010a) Apatite formation on bioactive calcium-silicate cements for dentistry affects surface topography and human marrow stromal cells proliferation. Dental Materials 26, 974-92.

Gandolfi MG, Van Landuyt K, Taddei P, Modena E, Van Meerbeek B, Prati C (2010b) Environmental scanning electron microscopy connected with energy dispersive $\mathrm{x}$-ray analysis and Raman techniques to study ProRoot mineral trioxide aggregate and calcium silicate cements in wet conditions and in real time. Journal of Endodontics 36, 851- 7.

Gu LS, Kim YK, Liu Yet al.(2011) Immobilization of a phosphonated analog of matrix phosphoproteins within cross-linked collagen as a templating mechanism for biomimetic mineralization. Acta Biomaterialia 7, 268-77.

Hasson D, Shemer H, Sher A (2011) State of the Art of Friendly "Green" Scale Control Inhibitors: A Review Article. Industrial \& Engineering Chemistry Research 50, 7601-7. 
Hoac B, Kiffer-Moreira T, Millán JL, McKee MD (2013) Polyphosphates inhibit extracellular matrix mineralization in MC3T3-E1 osteoblast cultures. Bone 53, 478-86.

Karan K, Yao X, Xu C, Wang Y (2009) Chemical profile of the dentin substrate in non-carious cervical lesions. Dental Materials 25, 1205-12.

Leonor IB, Balas F, Kawashita M, Reis RL, Kokubo T, Nakamura T (2009) Biomimetic apatite deposition on polymeric microspheres treated with a calcium silicate solution. Journal of Biomedical Materials Research B Applied Biomaterials 91, 239-47.

Li J, Yang J, Li J et al. (2013) Bioinspiredintrafibrillar mineralization of human dentine by PAMAM dendrimer. Biomaterials 34, 6738-47.

Liu Y, Li N, Qi Yet al. (2011a) The use of sodium trimetaphosphate as a biomimetic analog of matrix phosphoproteins for remineralization of artificial caries-like dentin. Dental Materials 27, 465-77.

Moon DH, Park JW, Cheong KH et al. (2013) Stabilization of lead and copper contaminated firing range soil using calcined oyster shells and fly ash. Environmental Geochemical Health 35, 705-14.

Omelon S, Georgiou J, Variola F, Mason N (2014) Dean Colocation and role of polyphosphates and alkaline phosphatase in apatite biomineralization of elasmobranch tesserae. Acta Biomaterialia 10, 3899-910.

Osorio R, Yamauti M, Sauro S, Watson TF, Toledano M (2012) Experimental resin cements containing bioactive fillers reduce MMPs mediated dentin collagen degradation. Journal of Endodontics 38, 1227-32.

Osorio R, Yamauti M, Sauro S, Watson TF, Toledano M (2014) Zinc incorporation improves biological activity of beta-tricalcium silicate resin-based cement. Journal of Endodontics 40, 1840-5.

Parirokh M, Torabinejad M (2010) Mineral Trioxide Aggregate: A Comprehensive Literature ReviewPart I: Chemical, Physical, and Antibacterial Properties. Journal of Endodontics 36, 16-27.

Rashchi F, Finch JA (2000) Polyphosphates: A review their chemistry and application with particular reference to mineral processing. Mineral Engineering 13,1019-35. 
Rezwan K, Chen QZ, Blaker JJ, Boccaccini AR (2006) Biodegradable and bioactive porous polymer/inorganic composite scaffolds for bone tissue engineering. Biomaterials 27, 3413-31.

Sauro S, Osorio R, Fulgêncio R et al. (2013) Remineralisation properties of innovative light-curable resin-based dental materials containing bioactive micro-fillers. Journal Materials Chemistry $B \mathbf{1}$, 2624-38.

Sauro S, Osorio R, Watson TF, Toledano M (2015) Influence of phosphoproteins' biomimetic analogs on remineralization of mineral-depleted resin-dentin interfaces created with ion-releasing resinbased systems. Dental Materials 31, 759-77.

Shen CY (1966) Alkaline hydrolysis of sodium trimetaphosphate in concentrated solutions and its role in built detergents. Industrial and Engineering Chemical Production and Research Development 5, 272-6.

Toledano M, Aguilera FS, Cabello I, Osorio R (2014a) Remineralization of mechanical loaded resindentin interface: a transitional and synchronized multistep process. Biomechanical Modelling and Mechanobiology 13, 1289-302.

Toledano M, Aguilera FS, Sauro S, Cabello I, Osorio E, Osorio R (2014b) Load cycling enhances bioactivity at the resin-dentin interface. Dental Materials 30, e169-188.

Wang C, Wang Y, Huffman NTet al. (2009) Confocal laser Raman microspectroscopy of biomineralization foci in UMR 106 osteoblastic cultures reveals temporally synchronized protein changes preceding and accompanying mineral crystal deposition. Journal of Biology and Chemistry 284, 7100-13.

Watson TF, Atmeh AR, Sajini S, Cook RJ, Festy F (2014) Present and future of glass-ionomers and calcium-silicate cements as bioactive materials in dentistry: Biophotonics-based interfacial analyses in health and disease. Dental Materials 30, 50-61.

Zhang Y, Wang Y (2013) Effect of application mode on interfacial morphology and chemistry between dentine and self-etch adhesives.Journal of Dentistry 41, 231-40. 
Table 1: Mineral to matrix ratio, relative presence of mineral and degree of crystallinity obtained for the different experimental groups, after Raman spectroscopy and cluster analysis.

\begin{tabular}{|c|c|c|c|c|c|c|c|c|}
\hline \multirow[b]{2}{*}{ Hybrid Layer } & \multicolumn{4}{|c|}{$24 \mathrm{~h}$} & \multicolumn{4}{|c|}{$1 \mathrm{~m}$} \\
\hline & $\begin{array}{c}\text { RPM } \\
961 \mathrm{~cm}^{-1}\end{array}$ & $\begin{array}{c}1,465 \\
\mathrm{~cm}^{-1}\end{array}$ & MMR & $\begin{array}{l}961 \mathrm{~cm}^{-1} \\
\text { FWMH }\end{array}$ & $\begin{array}{c}\text { RPM } \\
961 \mathrm{~cm}^{-1}\end{array}$ & $\begin{array}{c}1,465 \\
\mathrm{~cm}^{-1}\end{array}$ & MMR & $\begin{array}{l}961 \mathrm{~cm}^{-1} \\
\text { FWMH }\end{array}$ \\
\hline DW/BTCS-Zn & 22.29 & 13.26 & 1.68 & 38.74 & 18.57 & 11.89 & 1.56 & 20.52 \\
\hline PAS/BTCS-Zn & 16.16 & 22.34 & 0.72 & 35.33 & 41.06 & 7.61 & 5.40 & 45.72 \\
\hline PAS-TMP/BTCS -Zn & 13.45 & 10.85 & 1.24 & 35.53 & 16.87 & 6.62 & 2.55 & 36.73 \\
\hline
\end{tabular}

\begin{tabular}{|c|c|c|c|c|c|c|c|c|}
\hline \multirow{2}{*}{$\begin{array}{l}\text { Underlying } \\
\text { Dentine }\end{array}$} & \multicolumn{4}{|c|}{$24 \mathrm{~h}$} & \multicolumn{4}{|c|}{$1 \mathrm{~m}$} \\
\hline & $\begin{array}{c}\text { RPM } \\
961 \mathrm{~cm}^{-1} \\
\end{array}$ & $\begin{array}{c}1,465 \\
\mathrm{~cm}^{-1} \\
\end{array}$ & MMR & $\begin{array}{l}961 \mathrm{~cm}^{-1} \\
\text { FWMH }\end{array}$ & $\begin{array}{c}\text { RPM } \\
961 \mathrm{~cm}^{-1} \\
\end{array}$ & $\begin{array}{c}1,465 \\
\mathrm{~cm}^{-1} \\
\end{array}$ & MMR & $\begin{array}{l}961 \mathrm{~cm}^{-1} \\
\text { FWMH }\end{array}$ \\
\hline DW/BTCS-Zn & 37.86 & 8.60 & 4.40 & 35.51 & 45.75 & 4.55 & 10.05 & 15.93 \\
\hline PAS/BTCS-Zn & 56.94 & 7.81 & 7.29 & 28.93 & 64.01 & 6.19 & 10.34 & 38.52 \\
\hline PAS-TMP/BTCS -Zn & 43.03 & 6.70 & 6.42 & 32.30 & 47.86 & 4.87 & 9.83 & 35.50 \\
\hline
\end{tabular}

RPM: relative presence of mineral (phosphate peak $\left[961 \mathrm{~cm}^{-1}\right.$ ] height); MMR: mineral-to-matrix ratio (ratio of integrated areas of the phosphate $v 1-\mathrm{PO}_{4}{ }^{3-}$ symmetric stretch $\left[961 \mathrm{~cm}^{-1}\right]$ and contour of the $\mathrm{CH}_{2}$ deformation of the collagen $\left[1,465 \mathrm{~cm}^{-1}\right]$ ); and FWMH: degree of crystallinity (full width at half maximum of the phosphate band at $961 \mathrm{~cm}^{-1}$ ). 
Table 2: Mean and standard deviation (SD) of microtensile bond strength values, distribution of failure mode (\%) and pre-load failed specimens (\%) in each experimental group.

\begin{tabular}{|c|c|c|c|c|c|c|}
\hline & \multicolumn{3}{|c|}{$24 \mathrm{~h}$} & \multicolumn{3}{|c|}{$6 \mathrm{~m}$} \\
\hline & $\begin{array}{c}\text { Mean -Mpa- } \\
\text { (SD) }\end{array}$ & $\begin{array}{c}\mathrm{A} / \mathrm{M} / \mathrm{C} \\
\%\end{array}$ & $\begin{array}{c}\text { Tested/Failed } \\
\%\end{array}$ & $\begin{array}{c}\text { Mean -Mpa- } \\
\text { (SD) }\end{array}$ & $\begin{array}{c}\mathrm{A} / \mathrm{M} / \mathrm{C} \\
\%\end{array}$ & $\begin{array}{c}\text { Tested/Failed } \\
\% \\
\end{array}$ \\
\hline DW/BTCS-Zn & $34.1(3.1)^{\mathrm{A} 1}$ & $2 / 93 / 5$ & $95 / 5$ & $24.1(3.5)^{\mathrm{A} 2}$ & $10 / 90 / 0$ & $95 / 5$ \\
\hline PAS/BTCS-Zn & $33.3(7.2)^{A 1}$ & $2 / 90 / 8$ & $95 / 5$ & $34.0(3.8)^{B 1}$ & $3 / 97 / 0$ & $90 / 10$ \\
\hline PAS-TMP/BTCS -Zn & $28.2(3.1)^{\mathrm{A} 1}$ & $5 / 95 / 0$ & $95 / 5$ & $24.4(5.6)^{\mathrm{A} 1}$ & $5 / 95 / 0$ & $90 / 10$ \\
\hline
\end{tabular}

For each horizontal row: values with identical numbers indicate no significant differenceusing Student $t$ test $(p>0.01)$. For each vertical column: values with identical letters indicate no significant difference using Student-Newman-Keuls test ( $p>0.05)$. A/M/C: percentage of adhesive $(\mathbf{A})$, mixed $(\mathbf{M})$ or cohesive $(\mathbf{C})$ failures using a stereo microscope (magnification $\times 60$ ). 


\section{Figures}

Figure 1: Raman analysis of bonded interfaces at $24 \mathrm{~h}$ evaluation. DW/BTCS-Zn specimens are represented in $\mathbf{a}, \mathbf{b}$ and $\mathbf{c}$. PAS/BTCS-Zn specimens are shown in $\mathbf{d}$, $\mathbf{e}$ and $\mathbf{f}$ images, and PASTMP/BTCS-Zn bonded interfaces are displayed in $\mathbf{g}, \mathbf{h}$ and $\mathbf{i}$ graphs. $\mathbf{a}, \mathbf{d}$ and $\mathbf{g}$ are 2D micro-Raman maps of phosphate peak $\left(961 \mathrm{~cm}^{-1}\right)$ intensity at the dentine bonded interfaces. $\mathbf{b}$, e and $\mathbf{h}$ are $K$ means clustering maps of the Raman profile and $\mathbf{c}, \mathbf{f}$ and $\mathbf{i}$ are Raman spectra of principal components. At cluster images and Raman spectra adhesive is in violet, hybrid layer in blue, dentin is in green and red.

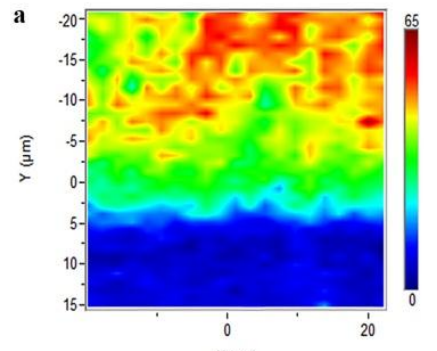

$\mathrm{X}(\mu \mathrm{m})$
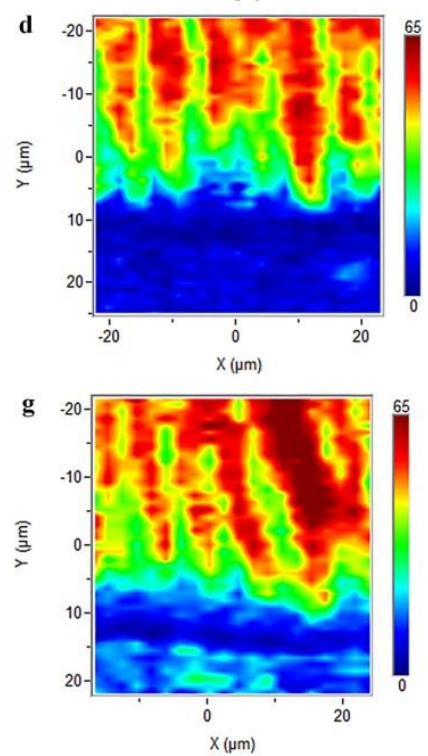

b
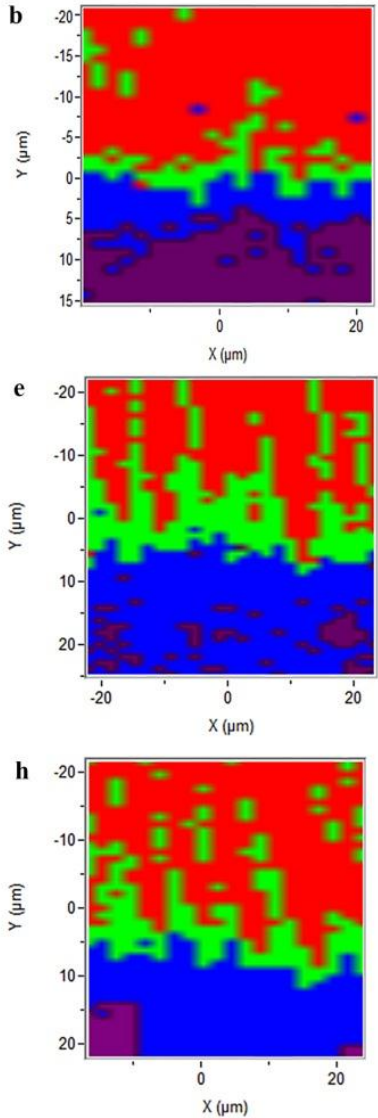
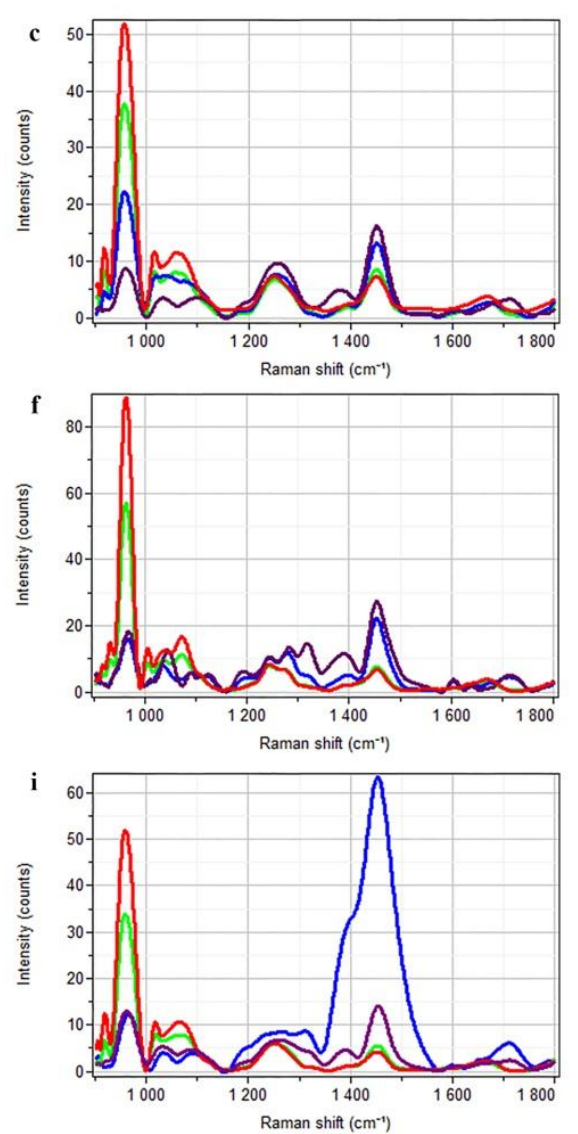
Figure 2: Raman analysis of bonded interfaces after $1 \mathrm{~m}$ storage in artificial saliva. DW/BTCS-Zn specimens are represented in $\mathbf{a}, \mathbf{b}$ and $\mathbf{c}$. PAS/BTCS-Zn specimens are shown in $\mathbf{d}, \mathbf{e}$ and $\mathbf{f}$ images, and PAS-TMP/BTCS-Zn bonded interfaces are displayed in $\mathbf{g}, \mathbf{h}$ and $\mathbf{i}$ graphs. $\mathbf{a}, \mathbf{d}$ and $\mathbf{g}$ are 2D microRaman maps of phosphate peak $\left(961 \mathrm{~cm}^{-1}\right)$ intensity at the dentine bonded interfaces. $\mathbf{b}, \mathbf{e}$ and $\mathbf{h}$ are $K$-means clustering maps of the Raman profile and $\mathbf{c}, \mathbf{f}$ and $\mathbf{i}$ are Raman spectra of principal components. At cluster images and Raman spectra adhesive is in violet, hybrid layer in blue, dentin is in green and red.
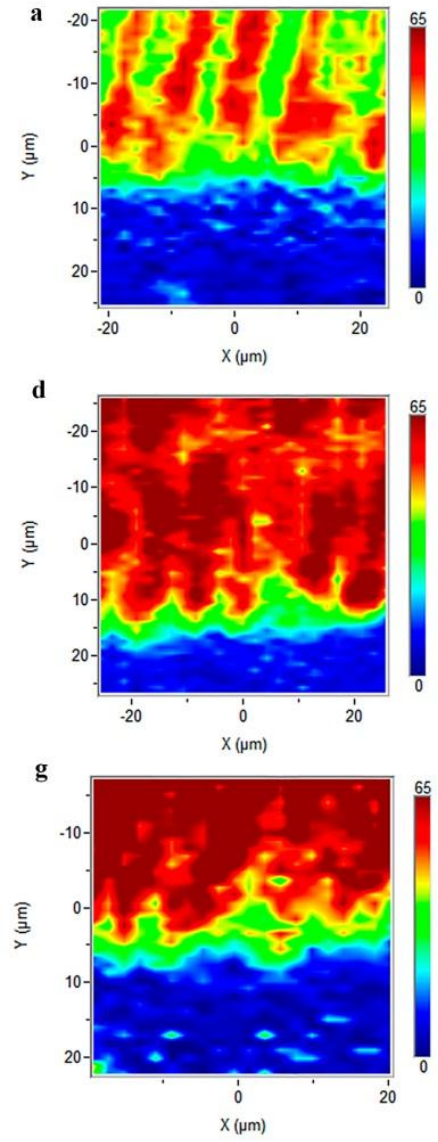
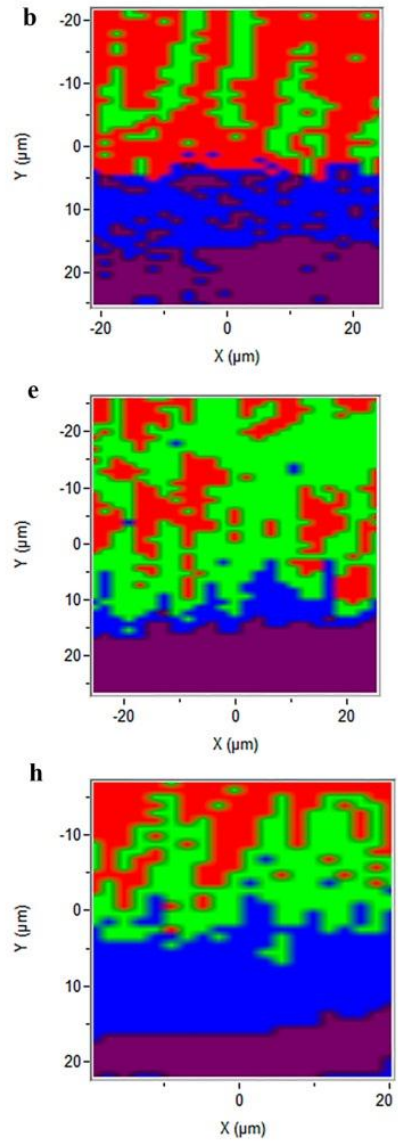
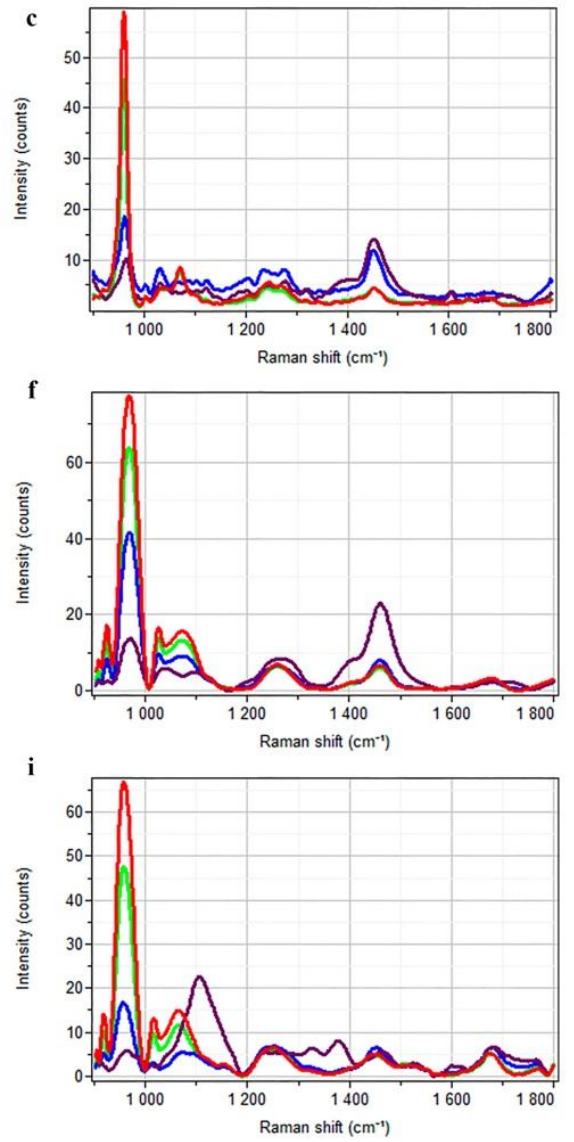
Figure 3: Scanning electron microscopy images of debonded resin-dentine specimens created using the different bonding approaches and tested after 6 months of artificial saliva storage.Images were taken at $3 \mathrm{kV}$ and a working distance of $15 \mathrm{~mm}$. a,b,c,d) Dentin side of a DW/BTCS-Zn specimen that failed in mixed mode. a) When the fracture is detected at the top of the hybrid layer, dentine is not visible and resin tags are fractured. b) If the fracture is located at the bottom of the hybrid layer, no mineral precipitation at the intertubular dentine is noticed; fractured resin tags are still present inside tubules. Demineralized collagen fibrils are shown. c,d) Crystal formations are detected. Globular formations are identified as silicon and calcium carbonate crystals (Ep1) and needles are Ettringite crystals that may also incorporate zinc (Ep2). e,f) Specimen from the PAS/BTCS-Zn group. It failed in adhesive mode within the hybrid layer, where it is not possible to see collagen fibrils, as result of mineral precipitation both at the intertubular and intratubular dentine. Fractured resin tags are still present in the dentine tubules. $\mathbf{g}$ ) Dentin side of a fractured specimen bonded with PASTMP/BTCS-Zn. Two different zones are observed: $\mathbf{h}$ ) fractured resin tags are presented protruding the dentine surface, and appeared completely covered by mineral formations; intertubular dentine is also covered by minerals and no demineralized collagen fibrils are evidenced; these zones are alternating with other in which collagen fibres are not resin covered or remineralized, even when some tags are inside the tubules i). 


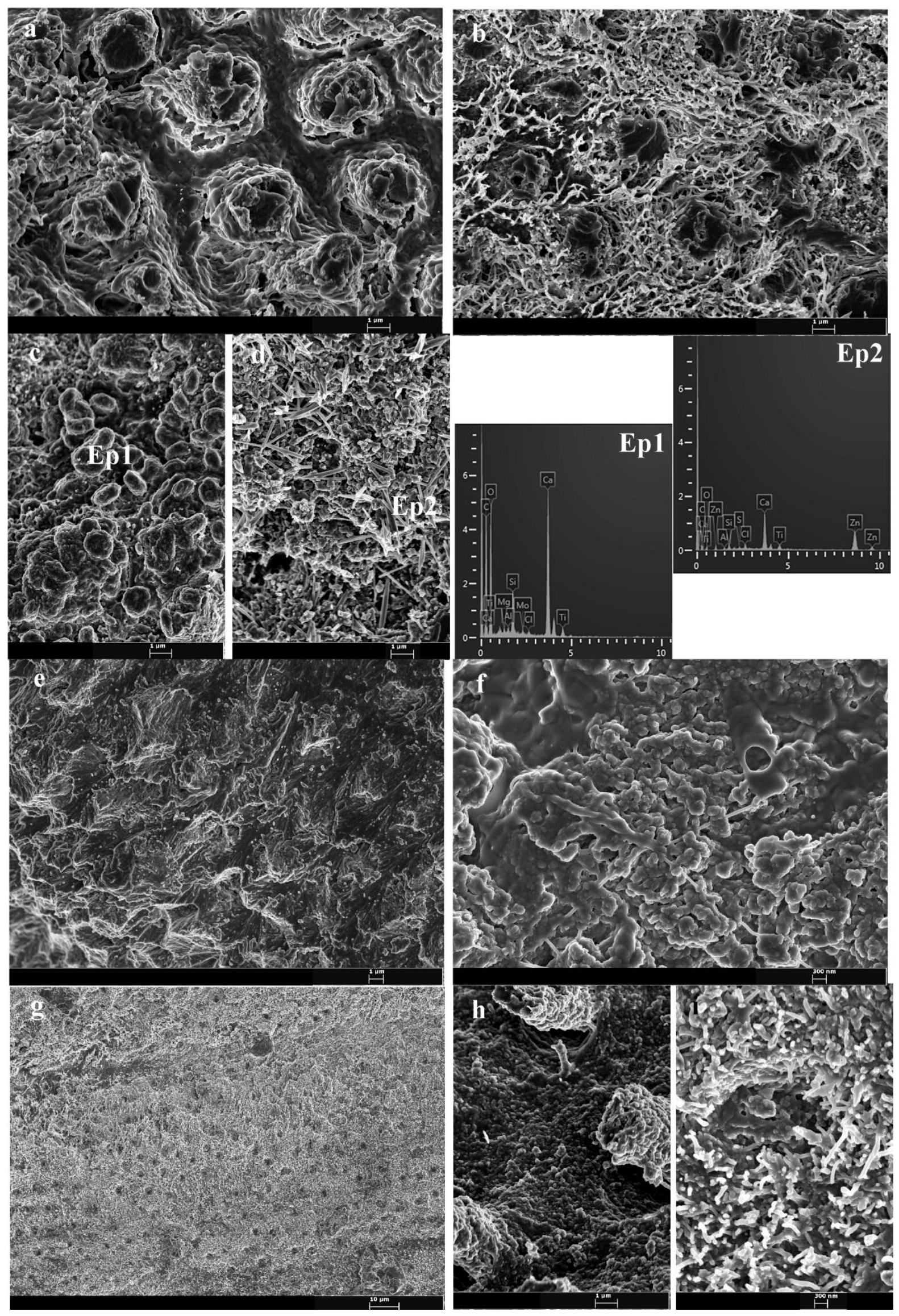

